\title{
Herb-Drug Interaction between Echinacea purpurea and Darunavir-Ritonavir in HIV-Infected Patients
}

\author{
José Moltó, ${ }^{1,2 *}$ Marta Valle, ${ }^{2,3}$ Cristina Miranda, ${ }^{1}$ Samandhy Cedeño, ${ }^{4}$ Eugenia Negredo, ${ }^{1}$ \\ Manuel José Barbanoj, ${ }^{2,3,5}$ and Bonaventura Clotet ${ }^{1,2,4}$ \\ Lluita contra la Sida Foundation, HIV Clinic, Hospital Universitari Germans Trias i Pujol, Badalona, Spain ${ }^{1}$ \\ Universitat Autónoma de Barcelona, Barcelona, Spain ${ }^{2}$; CIM-St Pau, Institut de Recerca HSCSP, Barcelona, Spain ${ }^{3}$; \\ IrsiCaixa Foundation, HIV Clinic, Hospital Universitari Germans Trias i Pujol, Badalona, Spain ${ }^{4}$; and \\ Clinical Pharmacology Department, Hospital de la Santa Creu i Sant Pau, Barcelona, Spain 5
}

Received 5 August 2010/Returned for modification 6 October 2010/Accepted 2 November 2010

\begin{abstract}
The aim of this open-label, fixed-sequence study was to investigate the potential of Echinacea purpurea, a commonly used botanical supplement, to interact with the boosted protease inhibitor darunavir-ritonavir. Fifteen HIV-infected patients receiving antiretroviral therapy including darunavir-ritonavir $(600 / 100 \mathrm{mg}$ twice daily) for at least 4 weeks were included. $E$. purpurea root extract capsules were added to the antiretroviral treatment $(500 \mathrm{mg}$ every $6 \mathrm{~h})$ from days 1 to 14 . Darunavir concentrations in plasma were determined by high-performance liquid chromatography immediately before and 1, 2, 4, 6, 8, 10, and $12 \mathrm{~h}$ after a morning dose of darunavir-ritonavir on days 0 (darunavir-ritonavir) and 14 (darunavir-ritonavir plus echinacea). Individual darunavir pharmacokinetic parameters were calculated by noncompartmental analysis and compared between days 0 and 14 with the geometric mean ratio (GMR) and its $90 \%$ confidence interval (CI). The median age was 49 (range, 43 to 67 ) years, and the body mass index was 24.2 (range, 18.7 to 27.5 ) $\mathrm{kg} / \mathrm{m}^{2}$. Echinacea was well tolerated, and all participants completed the study. The GMR for darunavir coadministered with echinacea relative to that for darunavir alone was $0.84(90 \% \mathrm{CI}, 0.63-1.12)$ for the concentration at the end of the dosing interval, $0.90(90 \% \mathrm{CI}, 0.74-1.10)$ for the area under the concentration-time curve from 0 to $12 \mathrm{~h}$, and $0.98(90 \%$ CI, 0.82-1.16) for the maximum concentration. In summary, coadministration of $E$. purpurea with darunavirritonavir was safe and well tolerated. Individual patients did show a decrease in darunavir concentrations, although this did not affect the overall darunavir or ritonavir pharmacokinetics. Although no dose adjustment is required, monitoring darunavir concentrations on an individual basis may give reassurance in this setting.
\end{abstract}

Despite the widespread use and proven effectiveness of highly active antiretroviral therapy (HAART) for the management of HIV infection, the use of herbal remedies and other types of complementary and alternative medicine (CAM) is common among HIV-infected patients, who often do not report such use to their doctor (14; J. Moltó, C. Miranda, S. Malo, M. Valle, A. Andreu, X. Bonafont, and B. Clotet, presented at the 11th International Workshop in Clinical Pharmacology of HIV Therapy, 2010). Many of these remedies are taken as complements rather than as substitutes for HAART. This, together with limited evidence to support the safety of many of these combinations, makes it possible for potentially significant interactions between certain types of CAM and antiretroviral drugs to occur.

Herbal medicines are often perceived to be innocuous and healthier than manufactured medicines even though they can act on the metabolism of antiretroviral agents, decreasing or increasing drug concentrations through induction or inhibition of metabolic pathways $(11,13)$. The most widely recognized botanical supplement associated with CYP450-mediated herbdrug interaction is St. John's wort (Hypericum perforatum). This herb may induce CYP3A4 activity, decreasing exposure to

\footnotetext{
* Corresponding author. Mailing address: Fundació Lluita contra la SIDA, Hospital Universitari Germans Trias i Pujol, Ctra de Canyet, s/n. 08916 Badalona. Barcelona. Spain. Phone: 349349788 87. Fax: 34 9346576 02. E-mail: jmolto@flsida.org.

${ }^{\nabla}$ Published ahead of print on 15 November 2010.
}

antiretroviral drugs such as indinavir or nevirapine $(6,18)$ and putting patients at risk of treatment failure and subsequent development of viral resistance to antiretroviral drugs. Clinical pharmacokinetic interaction studies between antiretroviral agents and botanicals nonetheless remain scarce in the literature.

Echinacea preparations rank among the top-selling botanical supplements worldwide $(1,4)$, and they are among the herbal medicines most commonly taken by HIV-infected patients (12), usually as a self-prescribed remedy and without medical supervision. Although the exact mechanism of action of echinacea remains unknown $(3,17,19,22,23)$, this substance has been reported to be an immunostimulant and its oral administration has been suggested to be beneficial in the early treatment of upper respiratory tract infections $(3,21,24)$.

The main constituents of echinacea preparations are caffeic acid derivatives and alkamides, which have a potential to interact with cytochrome P450 (1). Gorski et al. (10) investigated the in vivo effects of Echinacea purpurea on the activity of several CYP isozymes, including CYP3A4, after intravenous and oral midazolam administration. In that study echinacea selectively modulated the catalytic activity of CYP3A4 at both hepatic and intestinal sites. It inhibited intestinal lumen CYP3A4 activity, increasing the oral bioavailability of midazolam by nearly $50 \%$, but also induced hepatic CYP3A4 activity, significantly increasing the systemic clearance of the drug (10). This differential effect on CYP3A4 activity at two sites makes 
it difficult to predict potential effects on the disposition of other CYP3A4 substrates such as HIV protease inhibitors.

Darunavir is a protease inhibitor with demonstrated antiHIV efficacy in both antiretroviral-naïve and treatment-experienced patients $(5,16)$. Similarly to other protease inhibitors, darunavir undergoes extensive metabolism by the CYP3A4 isoform of the cytochrome P450 enzyme family (20). Therefore, in order to enhance its pharmacokinetic profile, darunavir must be coadministered with low doses of ritonavir, which acts as a potent CYP3A4 inhibitor, thereby boosting other CYP3A4 substrates. Thus, at the approved dosage of darunavir-ritonavir, darunavir concentrations at the end of the dosing interval remain well above the concentration needed to inhibit replication of HIV strains (20; V. Sekar, S. SpinosaGuzman, E. Lefebvre, and R. Hoetelmans, presented at the 16th International AIDS Conference, 2006). However, induction or inhibition of CYP3A4 activity by other agents might result in inadequately low or excessively high darunavir concentrations, respectively, compromising antiretroviral treatment effectiveness.

The aim of the present study was, therefore, to investigate the potential of this commonly used botanical supplement, $E$. purpurea, to interact with a protease inhibitor such as darunavir-ritonavir.

\section{MATERIALS AND METHODS}

Study design. This was an open-label, fixed-sequence study of 15 HIV-infected patients who were receiving antiretroviral therapy with darunavir-ritonavir at a dosage of 600/100 mg twice daily for at least 4 weeks and whose HIV-1 RNA load in plasma was $<50$ copies $/ \mathrm{ml}$. All patients gave written informed consent before enrollment, the protocol was approved by the ethics committee of our hospital, and the study was performed according to the stipulations of the Declaration of Helsinki (www.clinicaltrials.gov, NCT01046890).

Patients received capsules containing E. purpurea root extract at a dosage of $500 \mathrm{mg}$ every $6 \mathrm{~h}$ from days 1 to 14 (Arkocápsulas Echinácea, lot no. V05895B; Arkopharma, Madrid, Spain). Antiretroviral treatment remained unchanged.

Serial blood samples to determine darunavir and ritonavir concentrations in plasma were collected immediately before and 1, 2, 4, 6, 8, 10, and $12 \mathrm{~h}$ after a witnessed morning dose of darunavir-ritonavir on days 0 (darunavir-ritonavir) and 14 (darunavir-ritonavir plus echinacea).

Demographic and clinical variables, including age, body weight and height, and use of concomitant drugs, including over-the-counter medications, were recorded. Safety was evaluated by clinical interview and physical examination and by laboratory assessment (blood counts, chemistry, CD4 ${ }^{+} \mathrm{T}$-cell count, and HIV-1 RNA load) on days 0,14 , and 28 . To enhance adherence to scheduled clinical visits and the treatment protocol, patients were provided with a visit calendar. Apart from days 0 and 14, drug intake was not directly observed; adherence was assessed by means of a diary in which the patient recorded medication intake and by pill count on day 14 .

Analytical and pharmacokinetic analysis. Blood samples for darunavir and ritonavir determinations were collected into potassium- and EDTA-containing 10 -ml tubes. Plasma was isolated by centrifugation $(3,200 \times g$ for $15 \mathrm{~min})$, and stored at $-20^{\circ} \mathrm{C}$ until analysis. Darunavir and ritonavir concentrations were determined by high-performance liquid chromatography with a photo diode array detector (HPLC-PDA 2996; Waters, Barcelona, Spain), according to a validated method. The analytical column was a NovaPak $\mathrm{C}_{18}$ column (3.9 by 150 $\mathrm{mm}$ ) with a NovaPak $\mathrm{C}_{18}$ guard column (Waters). The method involved liquidliquid extraction of drug from plasma with methyl tert-butyl ether. The mobile phase consisted of a gradient elution with phosphate buffer in acetonitrile $(\mathrm{pH}$ 6.70). The method was linear over the range of 0.05 to $10.0 \mathrm{mg} / \mathrm{liter}$ for both drugs (the lower limit of quantification was $0.05 \mathrm{mg} /$ liter, and intra- and interday variation was $<10 \%$ ). Our laboratory subscribes to the external quality assurance program organized by the Association for Quality Assessment in Therapeutic Drug Monitoring and Clinical Toxicology of Radboud University Nijmegen Medical Centre, Nijmegen, Netherlands (7).

Darunavir and ritonavir pharmacokinetic parameters were calculated for each individual using a noncompartmental approach by means of Winnonlin (Version
2.0; Pharsight, Mountain View, CA). The area under the concentration-time curve during the dose interval $\left(\mathrm{AUC}_{\tau}\right)$ was calculated by means of the linear trapezoidal rule. Maximum concentrations $\left(C_{\max }\right)$ and the concentrations at the end of the dosing interval $\left(C_{\tau}\right)$ were obtained by inspection of the concentration data.

Statistical analysis. Data analysis was carried out using SPSS version 15.0 statistical software (Chicago, IL). Darunavir and ritonavir pharmacokinetic parameters were described by the geometric mean and compared between days 0 and 14 by the geometric mean ratio (GMR) and its $90 \%$ confidence interval (CI). Pharmacokinetic parameters were natural log transformed before analysis, and confidence intervals for means (and for the difference between two means) were constructed on the natural log scale based on an analysis of variance model with treatment as a fixed effect. The results were exponentiated and reported with lower and upper limits of the $90 \%$ CIs.

A power calculation indicated that 15 patients would provide an $80 \%$ chance of detecting a $40 \%$ difference in the $\mathrm{AUC}_{\tau}$ of darunavir at the $P=0.05$ level of significance.

\section{RESULTS}

A total of 15 Caucasian HIV-infected patients were included in the study. One patient was a woman, and three patients were coinfected with hepatitis $\mathrm{C}$ virus. The median age was 49 (range, 43 to 67 ) years, and the body mass index was 24.2 (range, 18.7 to 27.5 ) $\mathrm{kg} / \mathrm{m}^{2}$. In accordance with the inclusion criteria, all patients had an HIV-1 RNA load of $<50$ copies $/ \mathrm{ml}$. The median CD4 ${ }^{+}$T-cell count was 498 (range, 244 to 962 ) cells $/ \mathrm{mm}^{3}$.

Echinacea was well tolerated, and all participants completed the study. Reported adherence to echinacea and antiretroviral treatment was $100 \%$. No patient reported adverse events during the study, and all maintained an HIV-1 RNA load of $<50$ copies/ml at the end of the study.

Beside darunavir-ritonavir, antiretroviral treatment included lamivudine or emticitabine in 13 patients, raltegravir in 12, tenofovir in 11, and etravirine in 6 patients. Other antiretrovirals in use during the study included didanosine in three patients and abacavir and zidovudine in two patients and one patient, respectively.

Darunavir pharmacokinetics. Darunavir plasma concentration-time profiles following administration of darunavir-ritonavir alone or in combination with multiple doses of E. purpurea are shown in Fig. 1. Table 1 summarizes darunavir pharmacokinetic parameters with and without coadministration of echinacea. Coadministration of E. purpurea resulted in little change in darunavir pharmacokinetic parameters, with $C_{\tau}$ and $\mathrm{AUC}_{\tau}$ decreasing by averages of $16 \%$ and $10 \%$, respectively. However, looking at each participant individually, a decrease in darunavir $C_{\tau}$ or $\mathrm{AUC}_{\tau}$ by as much as $40 \%$ and $30 \%$, respectively, was observed in some patients (Table 1 and Fig. 2). There was no substantial effect on $C_{\text {max }}$.

Ritonavir pharmacokinetics. Figure 1 displays the ritonavir concentration-versus-time profile, and Table 2 lists the pharmacokinetic parameters of ritonavir after multiple-dose administration of darunavir-ritonavir alone or in combination with $E$. purpurea. Mean ritonavir pharmacokinetic parameters were essentially unchanged by coadministration of echinacea (Table 2).

\section{DISCUSSION}

There has been an upsurge in botanical supplement use in Western countries during the last decade, with $20 \%$ to $30 \%$ of 

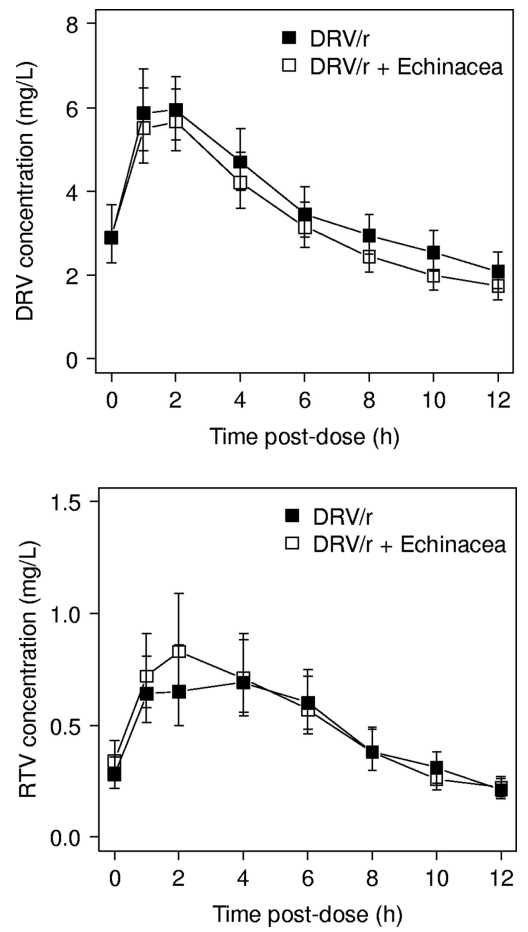

FIG. 1. Geometric mean darunavir (DRV) and ritonavir (RTV) plasma concentration profiles of darunavir-ritonavir (DRV/r) with or without coadministration of multiple doses of Echinacea purpurea. Error bars represent the $90 \%$ confidence interval.

prescription drug users taking concomitant herbal remedies (8, $25)$. The prevalence of herbal remedy intake is even higher among HIV-infected patients, and recent surveys indicate that more than one-third of HIV-infected patients may take herbal supplements along with antiretroviral therapy, often without notifying their physician (14; J. Moltó, C. Miranda, S. Malo, M. Valle, A. Andreu, X. Bonafont, and B. Clotet, presented at the 11th International Workshop in Clinical Pharmacology of HIV Therapy, 2010). In spite of this, studies of clinical pharmacokinetic interactions between antiretroviral agents and botanicals are scarce in the literature. The few pharmacokinetic studies that have been published have been conducted with healthy volunteers, and it has been noted that research has not kept pace with the standard of care for HIV management (13). As a result, it is difficult to extrapolate their results to HIVinfected patients receiving antiretroviral therapy with regimens commonly prescribed today, such as boosted protease inhibitors.
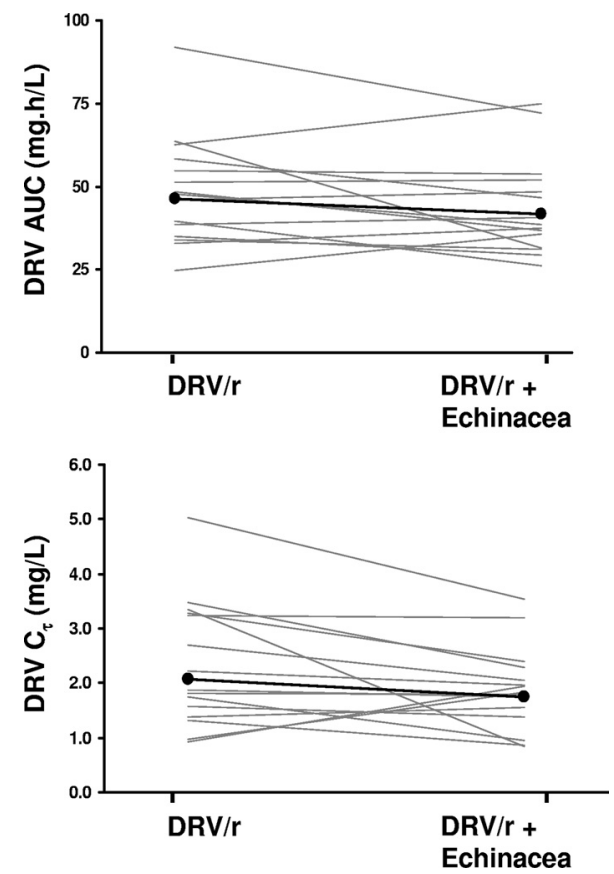

FIG. 2. Darunavir (DRV) concentration at the end of the dosing interval $\left(C_{\tau}\right)$ and area under the time-concentration curve $\left(\mathrm{AUC}_{\tau}\right)$ values after administration of darunavir-ritonavir with or without multiple doses of Echinacea purpurea. Gray lines represent individual values, and black lines represent the geometric mean.

In the present study we aimed to evaluate the potential interaction between one of the top-selling botanical supplements worldwide (1), E. purpurea, and darunavir-ritonavir as a model for boosted protease inhibitors. We hypothesized that there might be such an interaction based on previous in vivo data showing that E. purpurea influenced the activity of CYP3A4, the main enzyme involved in the metabolism of darunavir and other protease inhibitors, with opposite effects at the liver or at the intestinal sites (10). However, the effect of echinacea on darunavir pharmacokinetics $\left(C_{\tau}, \mathrm{AUC}_{\tau}\right.$, and $C_{\text {max }}$ ) was negligible in our study, as reflected in the close correspondence of the pharmacokinetic profiles in the presence or absence of coadministered echinacea across the entire 12-hour dosing interval. Nonetheless, although this result did not affect overall darunavir pharmacokinetics, individual patients did show a decrease in darunavir $C_{\tau}$ and $\mathrm{AUC}_{\tau}$ by as much as by $40 \%$ and $30 \%$, respectively, after coadministration of echinacea. This effect might be relevant in some clinical

TABLE 1. Comparison of darunavir pharmacokinetic parameters with and without coadministration of multiple doses of Echinacea purpurea ${ }^{a}$

\begin{tabular}{lccr}
\hline \multirow{2}{*}{ Parameter } & \multicolumn{2}{c}{ Result $^{b}$ for darunavir after administration of: } & GMR $(90 \%$ CI $)$ \\
\cline { 2 - 3 } & \multicolumn{1}{c}{ DRV/r } & DRV/r + echinacea & \\
\hline$C_{\tau}(\mathrm{mg} /$ liter $)$ & $2.1(1.7-2.6)$ & $1.7(1.4-2.1)$ & $0.84(0.63-1.12)$ \\
$\mathrm{AUC}_{\tau}(\mathrm{mg} \cdot \mathrm{h} /$ liter $)$ & $46.2(40.1-53.1)$ & $41.6(36.1-47.8)$ & $0.90(0.74-1.10)$ \\
$C_{\max }(\mathrm{mg} /$ liter $)$ & $6.4(5.6-7.2)$ & $6.2(5.5-7.1)$ & $0.98(0.82-1.16)$ \\
\hline
\end{tabular}

\footnotetext{
${ }^{a} \mathrm{DRV} / \mathrm{r}$, darunavir-ritonavir; GMR, geometric mean ratio; CI, confidence interval; $C_{\tau}$, concentration at the end of the dosing interval; $\mathrm{AUC}$, area under the time-concentration curve from 0 to $12 \mathrm{~h}$ after dosing; $C_{\max }$, maximum concentration.
}

${ }^{b}$ Data are expressed as geometric mean (90\% confidence interval). 
TABLE 2. Comparison of ritonavir pharmacokinetic parameters with and without coadministration of multiple doses of Echinacea purpurea ${ }^{a}$

\begin{tabular}{lccr}
\hline \multirow{2}{*}{ Parameter } & \multicolumn{2}{c}{ Result $^{b}$ for ritonavir after administration of: } & GMR (90\% CI) \\
\cline { 2 - 3 } & \multicolumn{1}{c}{ DRV/r } & DRV/r + echinacea & \\
\hline$C_{\tau}(\mathrm{mg} /$ liter $)$ & $0.21(0.17-0.26)$ & $0.22(0.18-0.27)$ & $1.04(0.60-1.41)$ \\
$\mathrm{AUC}_{\tau}(\mathrm{mg} \cdot \mathrm{h} / \mathrm{liter})$ & $6.16(5.03-7.55)$ & $6.44(5.26-7.89)$ & $1.05(0.78-1.39)$ \\
$C_{\max }(\mathrm{mg} /$ /iter $)$ & $0.86(0.69-1.08)$ & $1.01(0.81-1.27)$ & $1.17(0.85-1.61)$ \\
\hline
\end{tabular}

${ }^{a} \mathrm{DRV} / \mathrm{r}$, darunavir-ritonavir; GMR, geometric mean ratio; $\mathrm{CI}$, confidence interval; $C_{\tau}$, concentration at the end of the dosing interval; AUC ${ }_{\tau}$, area under the time-concentration curve from 0 to $12 \mathrm{~h}$ after dosing; $C_{\max }$, maximum concentration.

${ }^{b}$ Data are expressed as geometric mean (90\% confidence interval).

settings, such as in treatment-experienced patients with reduced viral susceptibility to darunavir. The variability in the effect of echinacea on darunavir pharmacokinetics we observed in this cohort may imply some degree of uncertainty in the CYP3A4 inductive/inhibitory potential of this herbal remedy. Considered together, these results support caution to be exercised and clinicians should be vigilant to the possibility of use of echinacea or other botanical supplements by their patients, monitoring plasma antiretroviral drug concentrations if possible.

The discrepancy between the lack of an interaction between echinacea and darunavir-ritonavir in our study and the increase in the oral bioavailability of the CYP3A4 substrate midazolam described by Gorski et al. (10) may be explained because patients in the present study were also receiving low doses of ritonavir, which is a potent CYP3A4 inhibitor. This inhibition of CYP3A4 by ritonavir could mask the influence of echinacea on CYP3A4 activity. If so, a remaining question is whether the lack of a significant interaction between boosted darunavir and echinacea that we report here would be reproducible in patients receiving other antiretroviral agents which are CYP3A4 substrates but not necessarily coadministered with ritonavir, such as nonnucleoside reverse transcriptase inhibitors or maraviroc.

Although echinacea is considered one of the safest herbal medicines, there has been some concern about its use in HIVinfected patients $(1,2)$, in whom it has been thought to be potentially dangerous based on its T-cell-stimulating effect, which might cause a rise in viral load as a result of increasing replication of infected $\mathrm{T}$ cells (15). In fact, some texts recommend that HIV-positive patients should avoid the use of echinacea. We found, however, that E. purpurea was well tolerated, with no evident safety issue of concern. All participants completed the treatment period, and all of them maintained an undetectable viral load at the end of the study.

One potential limitation of our study includes the fact that we did not perform independent phytochemical analyses for "marker compounds" in the E. purpurea product used in this study. With the multitude of Echinacea brands available on the market, there may be great variability in product content, formulation, dissolution, and bioavailability. Moreover, discrepancies between the labeled and the actual content of active constituents are possible, with commercial products not containing the labeled herb at all in some cases (9), thereby confounding the interpretation of pharmacokinetic drug interaction studies. To avoid this, a single lot of E. purpurea was purchased from a sole vendor. Arkopharma is a leader in the botanical supplement industry in Europe; it is externally controlled and has been granted the Good Manufacturing Practices certificate by the AFSSAPS (French Agency for the Sanitary Security for Health Products).

In summary, the results of this study indicate that coadministration of E. purpurea root with darunavir-ritonavir is generally safe and well tolerated in HIV-infected patients. Although the study did not show an effect on the overall darunavir or ritonavir pharmacokinetics, individual patients did show a decrease in darunavir concentrations. Although no dose adjustment is required, monitoring darunavir concentrations on an individual basis may give reassurance in this setting.

\section{ACKNOWLEDGMENTS}

We thank the staff at the clinical site where data were gathered for this study and the patients who participated. We also acknowledge the contribution of Mary Ellen Kerans, who gave her advice on English language expression in the final version of the manuscript.

We have no conflicts of interest that are directly relevant to the context of this study.

This study was funded by a grant from the Spanish Health Department of the Spanish Government (Ministerio de Sanidad y Política Social; TRA-084) and by the Lluita contra la SIDA Foundation. M.V. is supported by FIS (CP04/00121) from the Spanish Health Department in collaboration with l'Institut de Recerca de l'Hospital de la Santa Creu i Sant Pau, Barcelona, Spain, and is a member of CIBERSAM Network.

\section{REFERENCES}

1. Barnes, J., L. A. Anderson, S. Gibbons, and J. D. Phillipson. 2005. Echinacea species (Echinacea angustifolia (DC.) Hell., Echinacea pallida (Nutt.) Nutt., Echinacea purpurea (L.) Moench): a review of their chemistry, pharmacology and clinical properties. J. Pharm. Pharmacol. 57:929-954.

2. Barrett, B. 2003. Echinacea: a safety review. HerbalGram 57:36-39.

3. Barrett, B. 2003. Medicinal properties of Echinacea: a critical review. Phytomedicine 10:66-86.

4. Blumenthal, M. 1999. Herb market levels after five years of boom. HerbalGram 47:64-65.

5. Clotet, B., N. Bellos, J. M. Molina, D. Cooper, J. C. Goffard, A. Lazzarin, A. Wohrmann, C. Katlama, T. Wilkin, R. Haubrich, C. Cohen, C. Farthing, D. Jayaweera, M. Markowitz, P. Ruane, S. Spinosa-Guzman, and E. Lefebvre. 2007. Efficacy and safety of darunavir-ritonavir at week 48 in treatmentexperienced patients with HIV-1 infection in POWER 1 and 2: a pooled subgroup analysis of data from two randomised trials. Lancet 369:1169-1178.

6. de Maat, M. M., R. M. Hoetelmans, R. A. Mathôt, E. C. van Gorp, P. L. Meenhorst, J. W. Mulder, and J. H. Beijnen. 2001. Drug interaction between St John's wort and nevirapine. AIDS 15:420-421.

7. Droste, J. A., R. E. Aarnoutse, P. P. Koopmans, Y. A. Hekster, and D. M. Burger. 2003. Evaluation of antiretroviral drug measurements by an interlaboratory quality control program. J. Acquir. Immune. Defic. Syndr. 32: 287-291.

8. Eisenberg, D. M., R. B. Davis, S. L. Ettner, S. Appel, S. Wilkey, M. Van Rompay, and R. C. Kessler. 1998. Trends in alternative medicine use in the United States, 1990-1997: results of a follow-up national survey. JAMA 280:1569-1575.

9. Gilroy, C. M., J. F. Steiner, T. Byers, H. Shapiro, and W. Georgian. 2003. Echinacea and truth in labeling. Arch. Intern. Med. 163:699-704. 
10. Gorski, J. C., S. M. Huang, A. Pinto, M. A. Hamman, J. K. Hilligoss, N. A Zaheer, M. Desai, M. Miller, and S. D. Hall. 2004. The effect of echinacea (Echinacea purpurea root) on cytochrome P450 activity in vivo. Clin. Pharmacol. Ther. 75:89-100.

11. Izzo, A. A., and E. Ernst. 2009. Interactions between herbal medicines and prescribed drugs: an updated systematic review. Drugs 69:1777-1798.

12. Ladenheim, D., O. Horn, U. Werneke, M. Phillpot, A. Murungi, N. Theobald, and C. Orkin. 2008. Potential health risks of complementary alternative medicines in HIV patients. HIV Med. 9:653-659.

13. Lee, L. S., A. S. Andrade, and C. Flexner. 2006. Interactions between natural health products and antiretroviral drugs: pharmacokinetic and pharmacodynamic effects. Clin. Infect. Dis. 43:1052-1059.

14. Littlewood, R. A., and P. A. Vanable. 2008. Complementary and alternative medicine use among HIV-positive people: research synthesis and implications for HIV care. AIDS Care 20:1002-1018.

15. Luettig, B., C. Steinmuller, G. E. Gifford, H. Wagner, and M. L. LohmannMatthes. 1989. Macrophage activation by the polysaccharide arabinogalactan isolated from plant cell cultures of Echinacea purpurea. J. Natl. Cancer Inst. 81:669-675.

16. Mills, A. M., M. Nelson, D. Jayaweera, K. Ruxrungtham, I. Cassetti, P. M Girard, C. Workman, I. Dierynck, V. Sekar, C. V. Abeele, and L. Lavreys. 2009. Once-daily darunavir/ritonavir vs. lopinavir/ritonavir in treatment-naive, HIV-1-infected patients: 96-week analysis. AIDS 23:1679-1688.

17. Percival, S. S. 2000. Use of echinacea in medicine. Biochem. Pharmacol 60: $155-158$.
18. Piscitelli, S. C., A. H. Burstein, D. Chaitt, R. M. Alfaro, and J. Falloon. 2000 Indinavir concentrations and St John's wort. Lancet 355:547-548.

19. Rininger, J. A., S. Kickner, P. Chigurupati, A. McLean, and Z. Franck. 2000 Immunopharmacological activity of Echinacea preparations following simulated digestion on murine macrophages and human peripheral blood mononuclear cells. J. Leukoc. Biol. 68:503-510.

20. Rittweger, M., and K. Arasteh. 2007. Clinical pharmacokinetics of darunavir. Clin. Pharmacokinet. 46:739-756.

21. Shah, S. A., S. Sander, C. M. White, M. Rinaldi, and C. I. Coleman. 2007. Evaluation of echinacea for the prevention and treatment of the common cold: a meta-analysis. Lancet Infect. Dis. 7:473-480.

22. Sharma, M., J. T. Arnason, A. Burt, and J. B. Hudson. 2006. Echinacea extracts modulate the pattern of chemokine and cytokine secretion in rhinovirus-infected and uninfected epithelial cells. Phytother. Res. 20:147-152.

23. Sharma, M., J. T. Arnason, and J. B. Hudson. 2006. Echinacea extracts modulate the production of multiple transcription factors in uninfected cells and rhinovirus-infected cells. Phytother. Res. 20:1074-1079.

24. Sharma, S. M., M. Anderson, S. R. Schoop, and J. B. Hudson. 2010. Bactericidal and anti-inflammatory properties of a standardized Echinacea extract (Echinaforce): dual actions against respiratory bacteria. Phytomedicine 17:563-568.

25. Tindle, H. A., R. B. Davis, R. S. Phillips, and D. M. Eisenberg. 2005. Trends in use of complementary and alternative medicine by US adults: 1997-2002. Altern. Ther. Health Med. 11(1):42-49. 